\title{
Interpreting for EFL Speakers in Humanitarian Organizations (MSF- Jordan): Roles and Challenges
}

\author{
Raidah Al-Ramadan \\ Department of Translation, \\ Yarmouk University, Jordan
}

\section{Maram Mufeed Al-Abdullah}

Languages Center, Yarmouk University, Jordan

DOI: https://doi.org/10.36941/jesr-2020-0127

\section{Abstract}

This paper sheds light on the subjective experiences of interpreters who work with an international, medical humanitarian organization, namely MSF-Jordan. The paper seeks to explore the role/s that interpreters identify themselves as performing when working with MSF-Jordan, the challenges they encounter when working for such organizations where most of the officials are EFL speakers, and the strategies they adopt to overcome the challenges. The study uses a qualitative method through conducting interviews in the form of a questionnaire that was sent to seventeen interpreters who worked or still working with MSF-Jordan between 2011-2019. The questionnaire used in this study was developed by Stahuljak and its selection is based on its suitability for the purposes of the current study. Eleven interpreters responded, and the data was analyzed considering the research questions. The results show that interpreters identify themselves as performing the roles of conduits, culture brokers, and other. The interpreters identified challenges related to their lack of experience specifically in specialized terminologies, officials' accents, officials' speaking pace, their feel of lack of recognition, too much work and having additional tasks other than interpreting. The study further shows that interpreters opt for various strategies to overcome the challenges such as asking for help in relevance to lack of knowledge, asking officials to repeat, guessing, and asking the officials to write down what they say to overcome the impediment of EFL.

Keywords: interpreters, MSF-Jordan, EFL, roles, challenges, strategies

\section{Introduction}

Interpretation is occupying, nowadays, a very important position in almost all fields. Its importance is emerging in war zones, non-war zones, courts, peace negotiations, health care, diplomatic fields, and international organizations, among others. The need for interpreters is still urgent in some fields even though machine translation is nowadays emerging on the surface. However, the fact that machine translation is not yet considered fully reliable in sensitive contexts as in courts and politics creates a persistent need for human interpreters.

The research that investigates the roles played by interpreters and the position they hold in various fields is tremendous. There has been, however, a dichotomy among researchers regarding the 
role of the interpreter in general. The debate is whether the interpreter is a mere 'linguistic transferrer' that should have no 'voice' or existence in the situation involved or whether he/she is no longer a 'mere cipher' and that he/she has a voice that is heard in situations in which interpreting is involved (Morris, 2010, Hermans, 2010, Davidson, 2010).

Interpreters' roles in different fields have been a recent issue among translation scholars. Avery (2001) discusses the roles of interpreters in health care, where there is a persistent need for them. In the United States, for instance, immigrants speak more than three hundred different languages. These immigrants require health care and providing them with the required heath care entails the availability of interpreters of various language pairs. In this regard, Avery draws a distinction between two perspectives on how interpreters are viewed in medical fields: the 'neutral' versus the 'active' interpreter. By the neutral interpreter perspective, Avery refers to the medical interpreter as only 'message passing', where the presence of the ideal interpreter is 'unobtrusive' and 'non-relational'. The only responsibility of the medical interpreter, according to this perspective, is to provide precise and complete transmissions of the message; Interpreters are not encouraged to establish relationships with patients and are not expected to initiate any interventions of their own accord. In contrast to the neutral interpreter, the active interpreter perspective views the medical interpreter as a person of various responsibilities that go beyond that of message passing. The interpreter here is a 'social presence' that cannot be ignored, and he/she is expected to 'negotiate cultures' where their bilinguality adds more tasks on them (Avery 2001: 4-5).

In an article that investigates the roles of interpreters in the registration interviews which the UNHCR ran in Tirana, Albania after the war in 2000, Jacquement (2010) maintains that interpreters' roles have changed. The interpreter's prescribed role was to provide legal documentations to the asylum seekers and to mediate between the asylum seekers and humanitarian organizations. However, interpreters were found to be investigating the asylum seekers' claims and identifying false claims. In this respect, Jacquement criticizes the interpreters who acted as institutional aids and gatekeepers. The interpreters' competence, he adds, allowed them to control the flow of the interaction and pass judgment on the asylum-seeker being interviewed. However, the change of roles, sees Jacquement, is one of the technologies of power put up by humanitarian relief agencies to manage mass displacements of people. The UNHCR choice to deny the asylum seekers the right to tell their stories was viewed by the asylum seekers' applicants as a lack of "humanity" on the part of the organization.

Among other fields in which Translation Studies' researchers have studied interpreters' roles is legal court proceedings. Morris (2010) argues that recent legal systems require interpreters to function as 'faceless' voices where even their names may not appear in court records except when the person who is interpreted for is referred to as speaking through an interpreter. Morris further provides examples of the many images that legal actors have given to interpreters in legal proceedings including the interpreter as a conduit, a transmission belt, a translating machine, a transmission wire, a cipher, and a mouthpiece among others. All these images view interpreters as ones who only interpret without having any presence other than that of linguistic, and as being impartial. However, interpreters are recently becoming more aware of their roles, which go beyond that of linguistic, and as a result, these images and views are not well received by all interpreters. A growing body of research has reflected interpreters' awareness and has shown them as intrusive elements in legal proceedings. Morris examines several examples to show that interpreters of legal proceedings might adjust their behaviors to meet other professionals' expectations in their attempts to act professionally.

Davidson's (2010) vision of interpreters falls under Avery's second category in which interpreters perform roles different from the ones expected. Davidson argues that interpreters can never act as conduits or mere echoes and he provides examples on the roles that interpreters perform in institutional settings (in this case, it is US public and private hospitals). Davidson argues that interpreters tend to align themselves with institutions and act in ways that strengthen their voices. Analyzing medical interviews (interaction between physicians and patients) when an interpreter is present, as well as the institutional context, Davidson shows the interpreter performing as informational "gatekeeper" who has the priority of keeping the interview "on track" and the physician 
"on schedule". Davidson's analysis shows that interpreters tend to interpret selectively in patterned ways and they, sometimes, answer patients' questions without the knowledge of the physician, thus acting as "co-interviewers." However, Davidson views such actions from the side of interpreters as a result of time pressures that are put on them by the institution which requires them to keep interviews short and as scheduled. This, Davidson concludes, ends up with interpreters violating the ethics of the profession and acting in ways that are different from what is expected.

Like interpreters, translators are also researched in terms of their visibility or lack of visibility in the translated text. For Hermans (2010), the presence of translators in translated narratives is obvious. Even though translated fiction usually carries the name of the translator, the target readers are not usually meant to read it as a translation. In such a case, the translator's presence is hidden behind the narrating voice. Hermans points out that unlike interpreting, where the physical presence of the interpreter shows their involvement, the only thing we have in translated fiction is the translated text. However, the question of whether the translator's voice disappears in translated fiction is of importance to him. He argues that the translator's voice, or the "other" voice, as he refers to it, is forced to appear in three cases: cases when the translated text is oriented towards an "implied reader", cases of "selfreflexiveness", and cases of "contextual overdetermination." In relevance to the first case, Hermans states that translated fiction addresses audience that is linguistically and geographically different from that of the source text. As a result, the translator's voice interferes by providing information that are seen important to ensure communication with the new audience. The second case of "selfreflexiveness" or "self- referentiality" as he calls it, occurs when the source text includes features specific to a certain language such as wordplay or polysemy and other devices that might be challenging for the translator and thus force them to intervene using brackets or notes, which, in turn, remind the readers of the translator's presence. The third case of contextual overdetermination is used by Hermans to refer to situations in which the translator does not have much freedom because of the narrative context's constraints. To illustrate, Hermans provides the translator of Max Havelaar who, when faced with a main passage that required finding an equivalent proverb that consists of four words and each word had to begin with the letters $\mathrm{E}, \mathrm{H}, \mathrm{V}$, and $\mathrm{W}$, had no option but to provide lengthy 'Translator's Note'. However, the visibility of this 'second' voice or the 'other' voice in every case depends on the adopted 'translation strategy' and on the 'consistency with which it had been carried through' (Hermans 2010: 198).

The question that rises here is whether the presence of interpreters or translators breaks translation codes of ethics. Do translators think of codes of ethics when they act? In this regard, Dolmaya (2011) investigates seventeen ethical codes taken from profession-oriented networks in fifteen countries and compares them in terms of their common principles to stress gaps in the guidelines. Dolmaya compares the codes with issues that are debated in the forum of Ethics and Professionalism of TranslatorsCafe.com to study the applicability of these codes to ethical dilemmas that translators usually face in their practice. By the comparison, Dolmaya aims to show the most important values to profession-oriented translation networks. Her analysis shows that networks agreed on only two principles, namely confidentiality and competence. However, other principles relevant to impartiality, accuracy, conflict resolution, professional development, advertising, translator rights and working conditions are addressed by only some of the codes and their guidelines are occasionally in conflict. Dolmaya concludes that there is a lack of consensus on ethical translation behavior and that when the codes do not address certain aspects of the profession, translators are left with no guiding principles for ethical behavior. For Dolmaya, a translator is ethical in the view of profession-oriented translation networks when they abide by the principles laid out in the network's code of ethics, and whether translators are performing ethically when practicing the profession depends on what network they belong to.

The above review of literature shows that translators' neutrality might be influenced. Among the factors that influence interpreters' decisions, Baker \& Maier (2011:2-4) mention technological developments, social developments, and political developments. They argue that Translators have always been warned to be neutral and not to be engaged. However, political challenges and ideologies 
are found to be factors that affect their decisions.

Accordingly, it could be argued that there are situations in which interpreters, and sometimes against their own choices, break their neutrality and assume different roles or perform additional tasks other than the ones assigned to them. The current paper focuses on interpreters who work for an international organization and whose work involves interaction with refugees receiving healthcare in a host country. It seeks to see how interpreters view their work conditions, the roles they assume, the challenges they face and the strategies they adopt to overcome these challenges.

However, before discussing the notion of interpreting for international organizations, the effect of English as a lingua franca and its influence on interpretation is first discussed since English is the language of communication in international organizations under study even when the people working there are not native speakers of English.

\subsection{English as a Lingua Franca (ELF) and Interpretation}

Since there are more nonnative speakers of English than native speakers and the number is still growing, studying English as a lingua Franca is of importance. The term "lingua franca" refers to a common language used for communication between people with different first languages (Seidelhofer, 2005, p. 339). It is a language that is used in situations where it is acquired as a foreign language by the two interlocutors or where it is a native language of one of the interlocutors.

In using the term "Lingua Franca", Jennifer Jenkins (2009, pp.200-2001) refers to the specific communication context of English used as a language that speakers of different linguacultural backgrounds use when these speakers are more than the English speakers. Jenkins describes ELF as involving both common ground and local variation. For her, ELF speakers have a shared linguistic common ground just as that shared common ground found among the many varieties of English that is referred to as 'English as a native language' (ENL). Some linguistic forms found among ELF speakers are like the ones found among ENL speakers. However, other forms (that have arisen through contact between ELF speakers) are different. In this regard, Jenkins draws a distinction between two frequent and completely opposite reactions to ELF: the first is that ELF promotes monolithicity and the second is that ELF promotes pluricentricity. According to the first approach, ELF is monolithic and monocentric. i.e. as a "monomodal" where 'intercultural communication and cultural identity' are made as a necessary causality. The pluricentric approach views ELF as lacking standards, promoting too much diversity, and exhibiting errors as it departs from the Inner Circle Englishes (British and American); It is viewed as an approach in which 'anything goes' (202). According to the later approach, there is no distinction between learning English for intercultural communication in which native speakers of English may or may not be present (ELF) and learning English as a foreign language where English is learnt for communicating with English native speakers (EFL). (203)

In translation studies, researchers, such as Albl-Mikasa (2010) and Pym (2001), view the widespread use of English as a Lingua franca (ELF) a challenge to the profession of interpreting. AlblMikasa, on the one hand, raises the question of whether the interpreting profession would turn out to be 'redundant' and 'superfluous'. She explains that the spread of English as a 'dominating language' (using Casanova's terms) in cross- cultural communication is a threat to the profession of translation which might make the need for translators or interpreters vanish (127). She further adds that interpreters in the twenty first century have been obliged to change the 'lofty ideals' and positions they enjoyed in the twentieth century as a result of using English as a lingua franca (p.142). She further maintains that using English as a lingua franca has even impacted the performance of interpreters. Based on a cognitive-constructivist and functional view of language, she argues that non-native source text production, particularly nonnative accents affects the interpreter's comprehension process. Interpreters will have problems related to capacity in the comprehension process, in the form of accommodation and in the TL production process.

Pym (2001), on the other hand, sees translation market in continuous expansion at the same time in which English rises as international lingua franca. This expansion constitutes what he calls the 
'diversity paradox': while lingua franca appears to be reducing linguistic diversity, translation should be increasing it, and both are happening simultaneously.

In the same vein, Bassnett (2014) says that the twenty-first century has contributed to the benefit of translation, thanks to the mass movements of people for various reasons such as wars, persecution, or seeking better places, among others. These movements, argues Bassnett, led to encounters of languages making translation inevitable. In addition, the rapid rise of electronic media has increased consciousness of the importance of cross-cultural communication. For Bassett, the need for translation stems from the necessity of more awareness of cultural differences and to the huge need for intercultural understanding in the age of globalization.

Thus, it could be said that the need for human interpreting is not vanishing, at least for the near future. The fact that English is used by people of various backgrounds and native languages assures the need for interpretation or translation. As a lingua Franca, English is used to unite people, as in United Nations, conferences, and international organizations. However, translation or interpretation is unavoidable.

\subsection{Aims of the study}

This qualitative study seeks to present the subjective experiences of interpreters in providing in-person interpretations to refugees in healthcare. The study also seeks to explore the role/s that interpreters identify themselves as performing when working with humanitarian organizations (namely MSFJordan). It further seeks to explore the challenges that interpreters encounter when working for such organizations and the strategies they adopt to overcome such challenges. The focus of this paper is the interpreter who works for MSF-Jordan, an international humanitarian organization that provides medical help for Syrian refugees in Jordan as will be discussed in the following section.

\section{3 \\ MSF (Médecins Sans Frontières) or (Doctors Without Borders)}

MSF is an international, independent, medical, humanitarian organization. It is a 'not-for-profit organization' that was founded in Paris by doctors and journalists in 1971. 'Nowadays, MSF is worldwide movement of more than 42,0oo people with staff from over 150 countries. It has 19 national offices and an international office in Geneva, Switzerland' and is known around the world for their humanitarian services. The organization main aims include providing people, around the globe, who are affected by "armed conflict, epidemics, healthcare exclusion and natural disasters" with emergency aids. The organization offers help to all people regardless of their race, religion, gender or political views. The organization works in the name of the 'universal medical ethics'. They always provide help after disasters such as floods, earthquakes and wars (MSF activity report, 2011). When the Syrian war started in 2011, and the refugees began to flow out to the Jordanian borders, MSF organization rushed to provide them with the needed help.

MSF-Jordan operates three clinics in Irbid governorate, north of Jordan, that provide Syrian refugees with treatment for non-communicable diseases (NCDs). The three clinics offer medical care, home visits, as well as psychosocial support to nearly 5,00o patients with diseases such as diabetes and hypertension. MSF is also the main provider of reproductive health care for Syrians in north Jordan and provided surgical help for Syrian refugees in Ramtha city from 2011 till 2018 through its emergency project that offered emergency surgical and post-operative care to the patients who were wounded in the war. MSF also focuses on mental health care and offers support to Syrian children and their parents in the city of Mafraq. Their Reconstructive Surgery Hospital in Amman treats Syrian war-wounded patients where they are provided with orthopedic, reconstructive, and maxillofacial surgery including mental health support and physiotherapy.

The officials working for this organization are from different nationalities and backgrounds. To be able to perform their humanitarian work as desired, they needed interpreters to facilitate 
communication with refugees who speak Arabic knowing that the official language of the organization is English. Thus, MSF employ interpreters from the same region (Jordan and Syria) to communicate with the refugees (MSF website).

\subsection{Questions of the study}

The study aims at answering the following two questions:

1- What are the roles that interpreters of MSF-Jordan identify themselves as performing?

2- How do interpreters of MSF -Jordan view their interpreting experiences? What are the challenges they face? And how they overcome such challenges?

\subsection{Methodology}

The study used a questionnaire questions that were sent to 17 interpreters who worked or still working for MSF-Jordan in the period between 2011-2019. The questionnaire used in this study was developed by Zrinka Stahuljak in his paper 'War, translation, transnationalism: Interpreters in and of the war (Croatia, 191-1992)'. The choice of Stahuljak's questionnaire was based on its suitability for the purposes of the current study. The questionnaire consisted of twenty questions that aimed to show the nature of the interpreters' work (their roles), their impressions of the organization staff members they work with, the official's impressions toward the interpreters from their perspectives, the impediments interpreters encounter in communicating with the officials and the strategies they resort to when facing such challenges. The questionnaire was sent to the seventeen interpreters via email. However, only eleven interpreters answered the questions and emailed them back to the researchers. The data was then analyzed considering the research questions. Table (1) provides information of the interpreters who participated in the current study.

Table (1): Participants of the Current Study

\begin{tabular}{|c|c|c|c|c|c|c|c|c|c|c|}
\hline \multicolumn{11}{|c|}{ Participants } \\
\hline \multicolumn{2}{|c|}{ Gender } & \multicolumn{3}{|c|}{ Education } & \multicolumn{3}{|c|}{ Experience } & \multicolumn{3}{|c|}{ Experience with MSF } \\
\hline Male & Female & MA & BA & Other & $\leq 1$ & $\leq 5$ & $\leq 10$ & $\leq 1$ & $\leq 5$ & $\leq 10$ \\
\hline 4 & 7 & 6 & 4 & 1 & 1 & 8 & 2 & 5 & 6 & o \\
\hline \multicolumn{2}{|c|}{11} & \multicolumn{3}{|c|}{11} & \multicolumn{3}{|c|}{11} & \multicolumn{3}{|c|}{11} \\
\hline
\end{tabular}

\section{Discussion and Analysis}

\subsection{Roles}

The first question of the study is concerned with the roles that interpreters of MSF-Jordan identify themselves as performing. This question was answered by the interpreters with relevance to their responses on the nature of their work. The roles which MSF- Jordan's interpreters identified include information transfer (conduit), culture- broker, and other.

In translation theory, interpreters have been viewed as neutral conduits "through which words flow as if ideas and thoughts were shared among cultures" (Avery, 2001; María Gómez Amich, 2013, p.21). However, viewing the translator or the interpreter as a conduit has been disproved by other scholars such as Davidson (2010), who, from a communicative and participatory approach, sees that interpreters take on more responsibilities. Hence, for the communicative exchange to happen successfully, the interpreter is expected to have a cultural background in addition to mastering two languages. Interpreting for humanitarian organizations at times of conflict makes interpreting setting special as they witness suffering and mistrust. In such contexts, the interpreters' roles become more 
important for they not only decode linguistic differences but also cultural ones.

In the current study, nine of the eleven interpreters claimed that the conduit performance, which they expected as their main role, appeared to be one out of the several they assumed. However, they identified the conduit as their main role. The nine interpreters said they interpret everything to the patients and to the officials during the sessions, which normally last between $45-50$ minutes, without addition or omission. However, one interpreter said he, sometimes, does not translate everything the patient says out of sympathy. When asked about that, interpreter 8 said:

... because some of the patients curse and say inappropriate words to the expats when they are in pain. My humanity entails me to be biased in some cases with those people. Some of the patients do not have a bail "كالة" so they must be discharged to "Azz'tari camp". So here, I try to give more excuses than the patient says to help them stay more at the Hospital.

The interpreter here departs from his primary role, that is of the conduit, to that of the advocate. As a conduit, the interpreter is not allowed to do omissions, additions, or any changes. Acting as a patient advocate means stepping out of their professional role that requires impartiality and "message passing" using Avery's words. The concept of interpreters' advocacy is controversial as the extent to which the interpreter should be involved in the patient's needs is not agreed upon. The National Council on Interpreting in Health Care (NCIHC) defines advocacy as "Taking action or speaking up on behalf of a service user whose safety, health, well-being or human dignity is at risk with the purpose of preventing harm" (2005, p. 16). The given definition implies that advocacy can only take place in exceptional circumstances where the interpreter has no choice but to advocate. The reason claimed by interpreter 8 is stemmed from his sense of humanity. Taking the patient to the refugee camp, which is the interpreter's main reason for advocacy, might not be enough according to the definition as it is not contributing to the harm or risk of the refugee. However, knowing that the interpreter is the only one who is from Ramtha city, which is the border city with Syria, and where the hospital is located might be enough for the interpreter who shares the same culture, language, and religion with the refugee to advocate. Relating the interpreter's action to Baker and Maier's previously- mentioned argument about the factors that affect the decisions made by interpreters, we see a good example of the impact of ideology.

The other interpreters said they sympathize with the patients, but they still translate everything both parties say. Interpreter 1 said "I always felt great compassion and understanding towards my refugee brothers and sisters." To her, refugee patients are brothers and sisters. However, she interprets with no addition or omission. Three interpreters said they translate everything, but when they feel the patient is not telling the truth, they share this information with the officials after the session. Advocacy here is not for the patients; Rather, it is for the officials. The question of whether they interpret to the officials when they feel the patient is not telling the truth was answered by Interpreter 9 as "yes- I still interpret it, and I add to the official my personal impression at the end." In response to the same question, interpreter 3 says:

"I never change what they say just because I feel that it is not true, but I always share this with the expat psychologist after the session or during the session if I had the chance."

However, some interpreters choose not to share such information with the officials even if they feel the patient is not telling the truth such as interpreter 4 who says:

"No. I prefer to let the officials make their own conclusions regarding the overall credibility of the patient."

The notion of being biased to one party over another is clear in some of the interpreters' responses. However, some of them are caught between the patients and the officials and cannot decide who should receive their advocacy. Interpreter 8 says: 
...the most burdensome is that you do not know for whom you must be biased. The patients who might be friends and relatives? or the employer? the hardest job ever.

Interpreters seem to struggle with the desire to advocate for the refugee patients, especially when they witness situations in which the patients are unable to advocate for themselves. Interpreter 7 says:

I sympathize with them and feel very sad for the terrible circumstances they are going through. I sincerely hope that soon they will go back to their homes and live their lives peacefully.

Despite the feeling of sympathy towards the refugees, the interpreter says that he is, "unfortunately," obligated to neglect or suppress the feeling because it contradicts with the Organization's policy." Here the interpreter seems to abide by the ethical codes of the profession or the organization's policies.

From what has been discussed, it seems that the interpreters' narrative clearly shows that impartiality and neutrality required in any interpreting setting might not be easily carried out in conflict- related setting and is subject to change.

The second role identified by the interpreters is that of cultural- broker. The term cultural broker was coined within North American and cultural anthropology and was given to those who worked as linguistic, spiritual, and commercial negotiators or mediators (interpreters) between the Native Indians and the European colonizers back in the seventeenth and eighteenth centuries (Gustafsson and Norstroem, 2013, p. 4). Nowadays, the term is used in its wider context to refer to those who possess cross-cultural competence and strive to bridge the gap in multicultural contexts. Hale (2007, p. 45) sees that the notion of the interpreter as a cultural- broker emerged in health care interpreting where interpreters usually are not trained and are not aware of their role.

As a culture-broker, the interpreter is expected to speak the dialect of the patient and to have very good knowledge of the patients' cultural beliefs and practices. Acting as a culture-broker, the interpreter is also expected to meet with the officials before and after the session to discuss any observations. When the interpreter is conscious of possible misunderstandings in the communication between the care provider and the patient because of cultural differences, he/she acts as an educator for the care provider before and after the session.

Analyzing the data of the current study, all the interpreters said they provide help to the care providers when it comes to cultural differences and they advise them about it. Identifying this role, interpreter 3 says:

...I am more than an interpreter who conveys words. I am also a kind of a teacher for the expats team informing them about the culture, what is appropriate and what is not.

The interpreter views himself as a teacher who decides what is accepted and what is not in a certain culture. Interpreter 6 says:

Mostly, I stick to my role as an interpreter unless I give cultural advice or my own input about the understanding of the facial expressions or certain body gestures by the refugee that could be important for the session.

The role of cultural broker might sometimes be inconsistent with the interpreters' main role. However, it is unavoidable for meaning is usually encapsulated within cultural contexts and interpreters might be asked to help explain cultural differences. In this respect, interpreter 10 says:

I interpret what the patient says. Sometimes the expat asks about personal impressions for cultural and religion-related considerations.

The third role identified by the interpreters is that of other. Here, the term other is used to refer to tasks that may take the nature of administrative work, such as doing paperwork, arranging 
appointments, and contacting other parties, among others. Nine of the interpreters said they sometimes schedule appointments for patients, remind the staff with their appointments, and communicate with other NGOs in Jordan. One participant stated that she conducts interviews with the refugees and then she translates them and then converts them to numbers. Other tasks include preparing the room for the psychologist before the session. It should be noted, though, that such tasks are not very frequent as interpreting is identified as their major role. Interpreters 7 says:

Occasionally, I arrange meetings for some officials in the organization, but most of the time my tasks are strictly related to interpreting and translating.

Interpreter 3 says:

Beside interpreting, I am also responsible for calling beneficiaries to remind them of their appointments. I also prepare the room with the expat psychologist for the session. I am also responsible for written translation work (translating trainings, therapeutic sheets...)

\subsection{Interpreting Experiences and Challenges:}

The second question of the paper is concerned with the way interpreters view their interpreting experiences and the challenges they face. The first part of the question is related to the interpreters' perspectives toward their work experience with MSF-Jordan. In general, all interpreters say their work experience with MSF is good and the officials are cooperative and understanding. In this regard interpreter 3 says:

The best thing about my job is when I see a child improving because of our services. I feel super satisfied when I know that we are helping others. I would say the problems among team members is the worst thing at work. The most burdensome is when I have to interpret something for the team members that I know is not true.

However, interpreters of MSF-Jordan identified the following challenges: their lack of experience in specialized medical terminology, officials' poor English, officials' unclear English accents, lack of recognition, doing too much work, and performing additional tasks other than interpreting.

Six of the interpreters said that one of the main challenges they face is their lack of experience, particularly in specialized medical terminology. Having a look at table (1) which shows the participants' information, we notice that nine out of the eleven interpreters have less than five years of translation experience in general. The eleven interpreters have less than five years of experience with MSF, and one interpreter has no interpreting experience at all and his work with MSF organization is his first experience. Even though many of the participants have some translation experience, they seem to lack experience in the medical field. Interpreter 9, who is a physician with seven years of interpreting experience with humanitarian and international organizations, is hired to help other interpreters with specialized medical terminologies. Three interpreters said they overcome their lack of experience in specialized medical terminology by either asking officials' help, asking the physician interpreter, or searching the term via online dictionaries.

The second challenge the interpreters identify as facing is the officials' poor English and/or their unclear accents. As mentioned earlier, English is used as a lingua Franca in MSF and the people working there come from various countries and speak various native languages which results in various accents that might affect the interpreter's comprehension as argued earlier by Albl-Mikasa.

In this regard, Lin, Chang, \& Kuo (2013) argue that English is spoken with a variety of accents for being the popular lingua franca nowadays. 'Accent' is considered by interpreters as a 'frequent and serious' problem as it affects their listening comprehension' (Lin, et al 2013:31). When they interpret a spoken language, it is of importance for them to comprehend what is said to convey it in the expected manner. However, when their listening is subject to variety of English accents, their performance will 
be affected, and much pressure is added to their task. What adds to the problem here, is that both interpreters and officials are non- native speakers of the language, which causes difficulty in comprehension for both parties.

In the present study, five of the interpreters described the officials' English language as poor, and seven said their English accent is hard to understand. Ten interpreters view themselves as good EFL speakers for they have university degrees in English language or translation. However, they face difficulties interpreting for other EFL speakers. In this regard, interpreter 11 says:

Some expatriates do not speak English fluently, so it is sometimes difficult to understand what they mean. Also, some of them do not speak English clearly because their original language affects their accent.

In response to the same question, interpreter 1 says:

I remember facing a difficulty with one of the non-native English speakers when we were both working on a written translational work; they had some difficulty understanding what was written in proper English.

Interpreter 9, who is a physician, also views the officials' language as a challenge. In response to the question related to the challenges, he says:

Sometimes the level of their English, sometimes the accent they have, sometimes a word/expression that I do not know.

Regarding the same question, interpreter 3 says:

Yes, sometimes their poor English can be the cause of the miscommunication. For example, they are not able to understand everything said in English, or they cannot express their ideas in a clear way. It might be the cause of the miscommunication sometimes, mainly when they mention a theory in psychology that I am not familiar with. (To solve this, I always write notes and ask the national team about the concepts, or I search and learn).

Interpreters also mention the officials' speaking pace as another challenge. According to them, most officials speak very fast, which in addition to the foreign accent, make it difficult for them to understand what is said from the first time.

The interpreters who reported language mastery and accent as challenges mentioned certain strategies to overcome them. Among the strategies is asking the officials to repeat, paraphrase, elaborate, or write down what they say. In this regard, interpreter 8 says:

I ask them to repeat, and if I still have a difficulty understanding them, I try to guess what they intend to say in different words, I sometimes ask them to write what they say when the accent impedes me, and sometimes I ask" do you mean this ' or ' do you mean that'?

One of the interpreters said that the difficulty of accent is usually overcome by time when they get used to the officials' accents. However, officials only work for short periods of time (usually three months) and then they leave to be replaced by other EFL officials, and the interpreters then do their best to get used to the new officials' accents and so on.

The third challenge that some MSF Jordan interpreters identify as a challenge is their feel of lack of recognition. Four interpreters out of the eleven mentioned that they feel second in their work environment. Interpreter 3 says:

...what bothers me the most is two issues: first, when I feel that I am a secondary part of the team that does not have the same importance as that given to psychologists and councilors. For example, the psychologist or councilor is always informed of the sessions and trainings, but sometimes I am asked to interpret something suddenly as if there is no value for the nature of my work. The second issue is the misconception of the nature of my work. For example, an interpreter is only transferring words without 
considering the culture, the emotions, facial expressions etc...

Three interpreters described too much work as another challenge. They interpret many sessions each day, and each session lasts for 40-45 minutes. They usually have an average of five to six sessions per day plus meetings. In this regard, interpreter 5 says:

The worst thing about my job is the extreme mental exhaustion that I sometimes suffer from, especially on days where I do too many translation/ interpretation sessions.

\section{Conclusion}

The paper aimed to show how interpreters of MSF-Jordan view their interpreting experiences by defining the roles they perform and the challenges they face. The interpreters who participated in the study identify themselves as performing the roles of conduits, cultural brokers, and other. The study also aimed to show how interpreters of MSF reflected on their interpreting experiences with MSF in which English is spoken as a foreign language and identified the difficulties they face in their work. The interpreters identified challenges related to the officials' accents, their speaking pace, and their own lack of terminologies. However, the responses showed that when interpreters themselves lack good experience in the domain they work in, they face more challenges. Interpreters apply a variety of strategies to overcome the impediment of EFL, such as asking the officials to repeat what is said, guessing the intended word, and asking the officials to write down what they want to say among others mentioned earlier. Interpreters talked about their working environment as comfortable in general and that interpreting for EFL speakers might be easy whenever the EFL speakers use simple clear words and when they speak slowly. Interpreting for EFL speakers in this study required the interpreters to be facilitators of communication where they try to understand what is said and then convey it to the other party. They also mentioned they performed other tasks that made them act more than just interpreters. Again, interpreting for international medical organizations has its special context that should be studies more.

\section{References}

Albl-Mikasa, M. (2010). Global English and English as a lingua franca (ELF): Implications for the interpreting profession. Trans-kom, 32, 126-148.

Amich, M. G. (2013). The vital role of conflict interpreters. Nawa Journal of Language and Communication, 7(2), 1528.

Avery, M. P. B. (2001). The role of the health care interpreter: An evolving dialogue. Boston, National Council on Interpreting in Health Care (NCIHC).

Baker, M., \& Maier, C. (2011). Ethics in interpreter \& translator training: Critical perspectives. The Interpreter and Translator Trainer, 5(1), 1-14.

Bassnett, S. (2014). Translation studies. Routledge.

Davidson, B. (2010). "The interpreter as institutional gatekeeper: The social-linguistic role of interpreters in SpanishEnglish medical discourse". in Mona Baker (Ed.) Critical readings in translation studies (pp152-173). London; New York: Routledge.

Dolmaya, J. M. (2011). Moral ambiguity: Some shortcomings of professional codes of ethics for translators. The Journal of Specialised Translation, 15, 28-49.

Gustafsson, K., Norström, E., \& Fioretos, I. (2013). The interpreter-a cultural broker. Interpreting in a Changing Landscape, 187-202.

Hale, Sandra. 2007. Community Interpreting. Hampshire, New York: Palgrave Macmillan.

Hermans, T. (2010) 'The translator's voice in translated narratives', in Mona Baker (Ed.) Critical readings in translation studies (pp193-212). London; New York: Routledge.

Jacqument, M. (2010) 'The registration interview: Restricting refugees' narrative performance', in Mona Baker (Ed.) Critical readings in translation studies. (pp133-151).London; New York: Routledge.

Jenkins, J. (2009). English as a lingua franca: Interpretations and attitudes. World Englishes, 28(2), 200-207. 
Lin, I. H. I., Chang, F. L. A., \& Kuo, F. L. (2013). The impact of non-native accented English on rendition accuracy in simultaneous interpreting. Translation \& Interpreting, 5(2).

Morris, R. (2010). Images of the court interpreter professional identity, Role definition and self-image. Translation and Interpreting Studies 5(1), 20-40. John Benjamins Publishing Company.

MSF Activity report, 2011 Switzerland. Retrieved from http://www.msf.ch/fileadmin/msf/pdf/archives/msfch _activity_report/20120706_rep_annual_report_ocg_EN.pdf

National Council on Interpreting in Health Care. (2005). National standards of practice for interpreters in health care.

Pym, A. (2001). Translation and international institutions: Explaining the diversity paradox. presenté pendant l'atelier "Translation and Institutions" du congrès Language Study in Europe at the Turn of the Millenium, Societas Linguistica Europea, Katholieka Universiteit (Leuven, 28-31 août 2001).

Seidelhofer, B. (2011). Understanding English as a Lingua Franca. Oxford: Oxford University Press, 2011, 244 p. ISBN 978-0-19-437500-9

Stahuljak, Z. (2010) 'War, translation, transnationalism: Interpreters in and of the war (Croatia, 191-1992)', in Mona Baker (Ed.) Critical readings in translation studies. (pp 391-414)).London; New York: Routledge.

\section{Appendix: Interpreter Questionnaire}

1- How long have you been working for the organization?

2- What motivated you to work and join it?

3- What are your academic qualifications? And how much experience do you have?

4- Is there a specific person you interpret for? Or do you interpret for any of the officials?

5- How many times or how many sessions have you interpreted for them so far?

6- What is the average length of a session?

7- What are the impressions that are left by the organization officials for whom you are translating regarding your performance?

8- What are the impressions that are left by the patients regarding your job? Please try to describe, without naming, a person from each side who left the best and who left the worst impression on you. Generally speaking, what do you like the best about MSF- Holland officials? And what bothers you the most? Give an example for each.

9- What are the roles that you perform while working with the officials?

10- What difficulties do you face while interpreting for the officials who are not English natives? Do you think that their English is direct and clear? Or do you face difficulty in communicating with them?

11- In case you have difficulty in communicating with the officials, do you think that it is because they don't master English? Or is it because of your lack of experience? Provide examples if possible?

12- When having a difficulty in communication, what do you do? Do you ask them, for example, to repeat or use other words? Describe what strategies do you apply?

13- Do the officials feel that their English impede their work? Give example.

14- What do you think of your work conditions?

15- How do you feel toward the injured refugees?

16- Do you feel that your job requires you to help them? And that requires you to be more than an interpreter?

17- Do you translate everything they say for the officials?

18- When you feel that the patient is not telling the truth, do you interpret it for the officials?

19- What duties other than interpreting are assigned to you while at work?

20- What do you like the best about your job and what do you dislike the most? What is the most burdensome?

21- When at work, how do you feel in general and how do you feel while interpreting? 